\title{
A novel application of 3D printing technology facilitating shell wound healing of freshwater turtle
}

\author{
Tsung-Fu Hung ${ }^{1 *}$, Po-Jan $\mathrm{Kuo}^{2 *}$ and Yu-Shin $\mathrm{Nai}^{3 *}$ \\ 1 WeCare Animal Medical Center, 1F., No.270, Daxing Rd., Bade Dist., Taoyuan City 334, Taiwan; \\ roachbug@gmail.com \\ 2 School of Dentistry, Tri-Service General Hospital, National Defense Medical Center, Taipei, Taiwan; kuo- \\ pojan@gmail.com \\ 3 Department of Entomology, National Chung Hsing University, Taichung City, 402, Taiwan; \\ ysnai@nchu.edu.tw \\ * Correspondence: Tsung-Fu Hung: roachbug@gmail.com; Po-Jan Kuo: kuopojan@gmail.com; Yu-Shin Nai: \\ ysnai@nchu.edu.tw
}

Simple Summary: This report describes how to apply the combination of 3D scanning, computeraid design (CAD), and 3D printing to make a protective device for rescuing wild animals. In the past years, although the 3D tools have become relatively low-cost and reachable, the veterinary medical application based on this technology is quite limited. The presenting article successfully extricates a wild freshwater turtle from an extensive defect of the shell within a short period. Integration of multiple sciences to 3D technology can provide a facile model for veterinary medical applications.

\begin{abstract}
Numerous cases and a shortage of resources usually limit wild animal rescue. New technology implemented might save these severely injured wild animals from the situation of euthanasia by easing the requirement of intensive medication. Three-dimensional (3D) technologies provide precise and accurate results that improve the quality of the medical application. These 3D tools have become relatively low-cost and accessible in the past years. In the medical field of exotic animal, turtle shell defect is highly challenging because of inevitable water immersion. This report is the first attempt to apply the combination of 3D scanning, computer-aid design (CAD), and 3D printing to make a protective device that frees the wound from exposure to water or infection sources. The presenting techniques successfully extricate a wild freshwater turtle from an extensive shell defect within a short period. Integration of multiple sciences to 3D technology can provide a facile model for veterinary medical applications.
\end{abstract}

Keywords: 3D printing technology; freshwater turtle; Ocadia Spp.; shell wound healing

\section{Introduction}

Three-dimensional (3D) technology has been utilized in many medical applications due to its high precision and accuracy [1-3]. Recently, based on the development of 3D fabricating and medical imaging technology, increasingly affordable 3D printing technologies now make it possible to create highly customizable patient-tailored products [4].

In exotic animal medicine, shell trauma is a frequent casualty for wildlife turtles and tortoises [5-7]. The shell fractures were usually restored by external fixation utilizing orthopedic screws, orthopedic pins, surgical wiring, epoxy , or super glue to bridge fracture segments [8-11]. Those procedures provide instant protection and fixation of shell fracture $[5,7]$. However, a large shell defectinvolving the shoulder, pelvic area, or penetrating punctures of the coelom could lead to a poor or even grave prognosis [7]. In the case of missing fragments, the shell usually cannot be stabilized and rehabilitated, whichmay be fatal without adequate medication and intensive nursing. Keeping the wound moist and clean should be ideal for wound healing $[5,7,11]$. Although reptiles' wound healing is 
relatively slow compared to mammals, extensive shell loss becomes harsher and challenging for freshwater turtles since the inevitable water immersion and fecal contamination may cause recurrent infections and could procrastinate wound healing. Therefore, prolonged and intensive nursing becomes a medical burden[7].

Herein, we describe a feasible way to facilitate a wild freshwater turtle recovered from the delayed healing of severe and extensive shell defects, which reduced the intensity and frequency of medical care. A computer-aid design (CAD) device was fabricated by a fused deposition modeling (FDM) 3D printer, which provided physical support of defective shell structure. It protected the delayed healing wound from water exposure. After the delivery of the device, the burden of intensive care was drastically decreased, and the wound infection eased rapidly, which favored wound healing. We tracked down the wound condition in the 5th month, and one year after the delivery of the device, the follow-up results showed a favorable prognosis and were free from adoption without special care. This study demonstrates the novel attempts to apply 3D printing technology on the wild freshwater turtle for wound healing; the technique provides an alternative option for exotic animal medicine.

\section{Materials and Methods}

Animal Background

A female freshwater turtle (Ocadia Spp.) was found and rescued by a local wildlife rescue association in Taiwan. This $30 \mathrm{~cm}$ long turtle was found to have a severe shell defect on the left side (Fig. 1A). The coelomic membrane was exposed on the fracture site, and debris, dirt, and fly larva around the affected area. After two months of wet-to-dry docking[7] and supportive treatment with irrigating, flushing, and nutrition, the wound remained with minor infection, and the healing was delayed.

\section{Diagnosis and Treatment Plan}

The turtle presented with a $13 \mathrm{~cm}$ long oval shell lost on the left side involving carapace, plastron and most part of bridge (Fig. 1A). The left anterior edge of shell bridge was lost thus the support structure between carapace and plastron was disconnected. A fistula wound with pus discharge was found on the caudal side of the coelomic membrane, and several minor wounds spread around on the membrane with purulent discharge. Systemic infection was found according to the raised quantity of white blood cells. The wound on the exposed choelomic membrane was not healing well with recurrent infections after 2 months intensive care.

The daily care procedure included removing the dirty bandage, wound irrigation, nutrition support which may require a team of at least two people to complete. Moreover, the dressing was not waterproof to keep the defect free of water. Therefore, the wound was hard to maintain clean and reduce possible bacterial infections at the wound site for this freshwater turtle, especially when there was a shortage of human medical resources. The novel device as the customized shell was designed and fabricated following the flow chart (Figure 1) to provide mechanical support and water isolation.

\section{The Bone Model and Surface Model of the Defect Reconstruction}

The turtle underwent cone-beam computed-tomography (CBCT) (Newtom 5G, Italy) scanning (Fig. 1B left). The CBCT scan was obtained with $0.3 \mathrm{~mm}$ voxel size, power of 110 $\mathrm{kVp}, 5 \mathrm{~mA}, 10$ seconds exposure, and all the raw data were compacted in DICOM format for further radiographic diagnosis. The skeletal 3D model was reconstructed after adjusting the density until identified the tissue border was clearly in the software. (OsiriX MD, Pixmeo, Geneva, Switzerland). The 3D reconstructed model was exported as a stereolithography (STL) file for onward editing. The surface model of the defect was acquired by a structured-light 3D surface scanner (Einscan-S, Shining 3D, China) (Fig. 1B right) and exported as an STL file. All the 3D models were edited by Blender, a computer-aided design software (Blender, Blender Foundation, Dutch). The bone and surface models were 
carefully aligned and merged to show the locale of the bone edge under the scutum for confirming any bone degeneration in case of underlying infection.

The bone model was mainly used for diagnosis and the skeletal structure analysis, which indicated the mechanical force support area. Moreover, the surface model provides the detailed architecture and morphology of the defect and surrounding shell structure for the device outline design. The edited models were processed by slicer software (Kisslicer, Jonathan Dummer; Simplify3D, Simplify3D Inc., USA) and printed by a fused deposition modeling (FDM) type Delta 3D printer (Atom2, ALT, Taiwan) with modified polylactic acid (PLA) filaments (Gypsum, Next Print, Taiwan).

\section{Design, Construction and Fitting of the Protective Device}

The shell defect formed several undercuts on the carapace, plastron, and caudal part of a bridge, which would facilitate the fixation; however, the lost anterior edge of the skeletal bridge was a challenge for waterproof due to the unstable soft tissue while left forelimb movement. According to the reconstructed model, we designed a protective device (Fig. 1C) and printed (Fig. 1D) scribed previously. The device practicability was tested through fixation stability (Fig. 1E), not interfering animal activity, and waterproofing (Fig. 1F). A rim was designed and fabricated with a snap-fit groove to tightly engage the overhanging shell's undercuts (Fig. 2A). The junction between shell edge/soft tissue and the rim was diminished, and the groove of the rim was covered over the edge of the defect for at least $5 \mathrm{~mm}$ to achieve secure fixation. The prototype of the rim showed a promising engagement to the printed defect model (Fig.2B). After fixation, the rim was further remodeled to form a hull shape, with an opening kept above (Fig. 2C). The rim with hull structure was printed and tested again on the defect for stability (Fig. 2D). To reduce the chance of leakage, the junction between the rim groove and shell defect edge/soft tissue was filled by polydimethylsiloxane (PDMS) as sealant (Sylgard 184, Dow Corning, USA). A layer of silicone sealant (Silicone sealant, Dow Corning, USA)was applied to the outer surface between the shell and rim. Furthermore, a cover part was designed with a porthole to attach to the opening (Fig. 2E), and it was printed and secured on the opening of the hull structure (Fig. 2F). The edge of the cover, which contacts a layer of silicone sealant, coated the opening to close the opening waterproof. The porthole was designed with thread to fit a wide-mouth bottle cap to facilitate daily care.

\section{Daily Care and Wound Management}

The hull and cover were fixed as designed and would not be removed during daily care. The porthole was closed with the cap when the turtle was immersed in the water. The caudal pocket wound of the defect was cleaned daily and debrided through the porthole.

\section{Results}

\subsubsection{Device Fitting and Leakage Test}

The delivery of rim structure attested to the feasibility of non-invasion fixation. The rim was adapted to the defect and let the turtle stay in a room for 3 hours. The fitness of the rim was well-adapted, and the shape of the rim with hull structure was modified to slim (Fig. 2D and E) while the original design of the rim was too thick that might scratch and be pushed by the turtle's limbs. The hull structure was installed and rechecked as described above. The device attached to the defect was stable and did not reachable by animal activity. After delivery, animal behavior presented no difference, including scratching, constant hiding, unwillingness to move, and activity change. Clean gauze was placed in the hull without the cover, and the patient was half-immersed and constrained in water. After 1 hour, the gauze was checked as dry, indicating no water leakage into the hull.

\subsubsection{Patient Follow Up}


In the clinical observation, the affected area presented debris without applying the device to protect the defect. The exudate formation and chronic infection were not subsided at this stage (Fig. 3A). However, the protective device can cover the defect and avoid wound contamination during the healing process. The keratinization was presented on the coelomic membrane (Fig. 3B), and the counts of white blood cells were presented at normal range $\left(1.4 \times 10^{4} / \mu \mathrm{L}\right)$ at one-month recall after device application. The matured keratinized tissue was almost covered the defect surface at five months of recheck examination, and the wound was solid during palpation. (Fig. $3 \mathrm{C}$ ) At one-month recall, the tissue damage on the posterior defect border was healed without exudate discharge, and a leather-like granulation bed was in place. (Fig. 3D-F).

Using the CBCT 3D model accessed the hard tissue formation, the bony bridge development was observed on the fracture site of the anterior area. (Fig. 4A and D) Ossification was presented on the border and the central location of the defect. (Fig. 4B and E) Moreover, the fenestration lesion on the posterior side was reduced (Fig. 4C and F).3.2. Figures

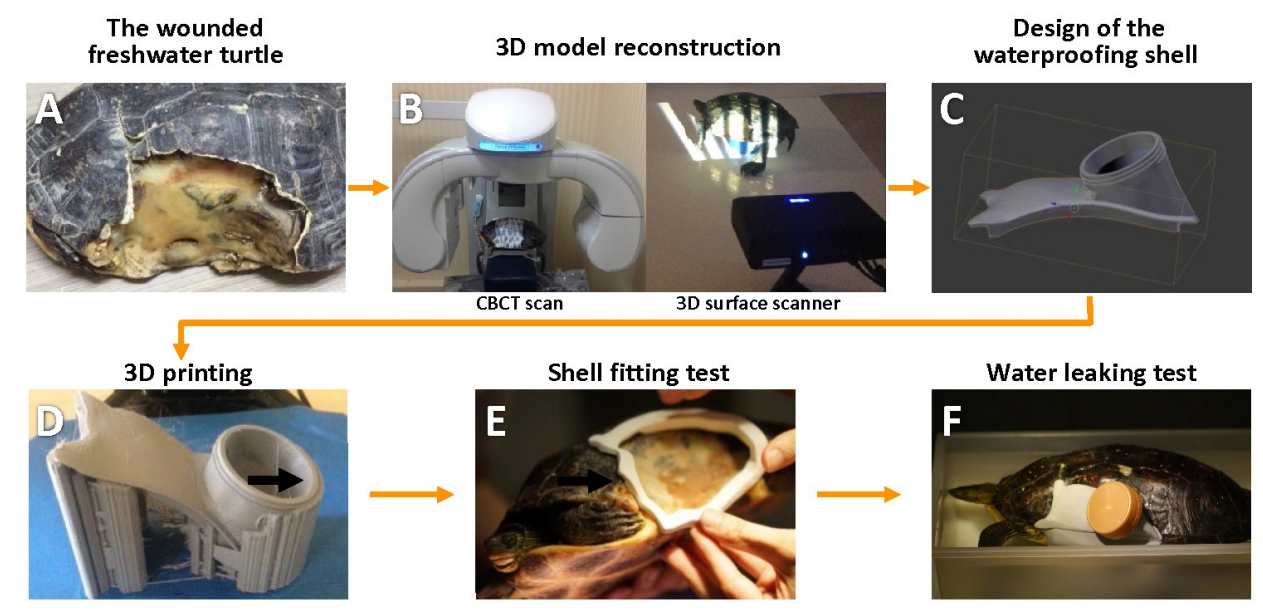

Figure 1. Flowchart of the treatment plan. (A) The female freshwater turtle (Ocadia sp.) with a $13 \mathrm{~cm}$ long oval defect; (B) The wounded freshwater turtle is scanned by CBCT and 3D surface scanner for reconstructing of 3D model ; (C) The reconstruction of 3D model of hard tissue and defect surface model are applied to design a waterproofing shell; (D) All the 3D model of the waterproofing shell are used to fabricate the template by a fused deposition modeling (FDM) type Delta 3D print; (E) Waterproofing shell fitting test is performed for secure fixation; (F) Water leaking test. 

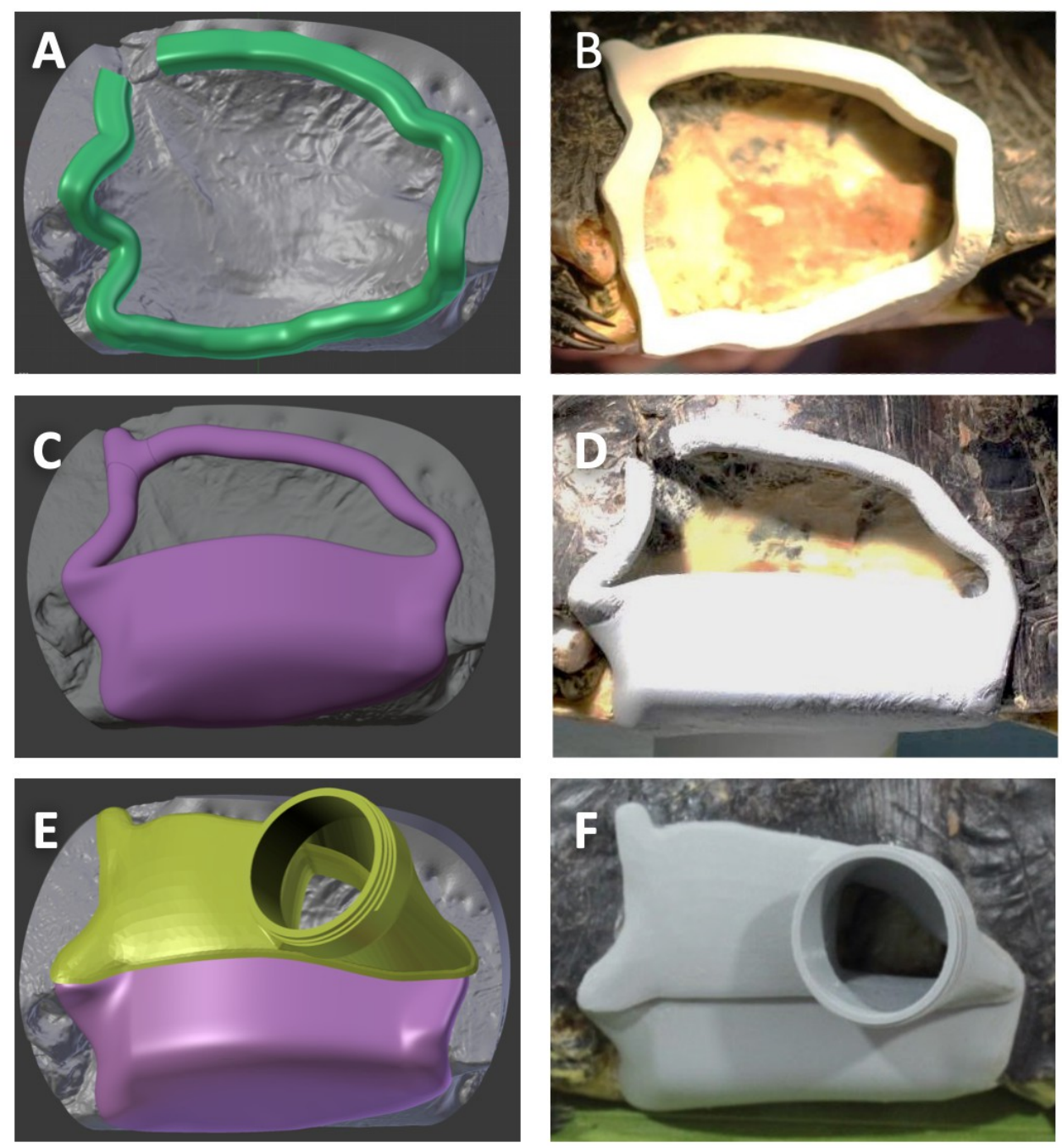

Figure 2. Design and fitting of the protective device on the defect of shell. 3D reconstructed models of protective device: (A) The CAD model of Snap-fit joints rim structure (green part); (B) printed and fitting tested on the defect; (C) A Hull structure (purple part) was designed according to the previous designed rim structure; (D) printed and fitting tested again on the defect; (E) A model of cover with a threaded porthole (yellow part) was designed; (F) printed and installed on the hull structure for further test. 

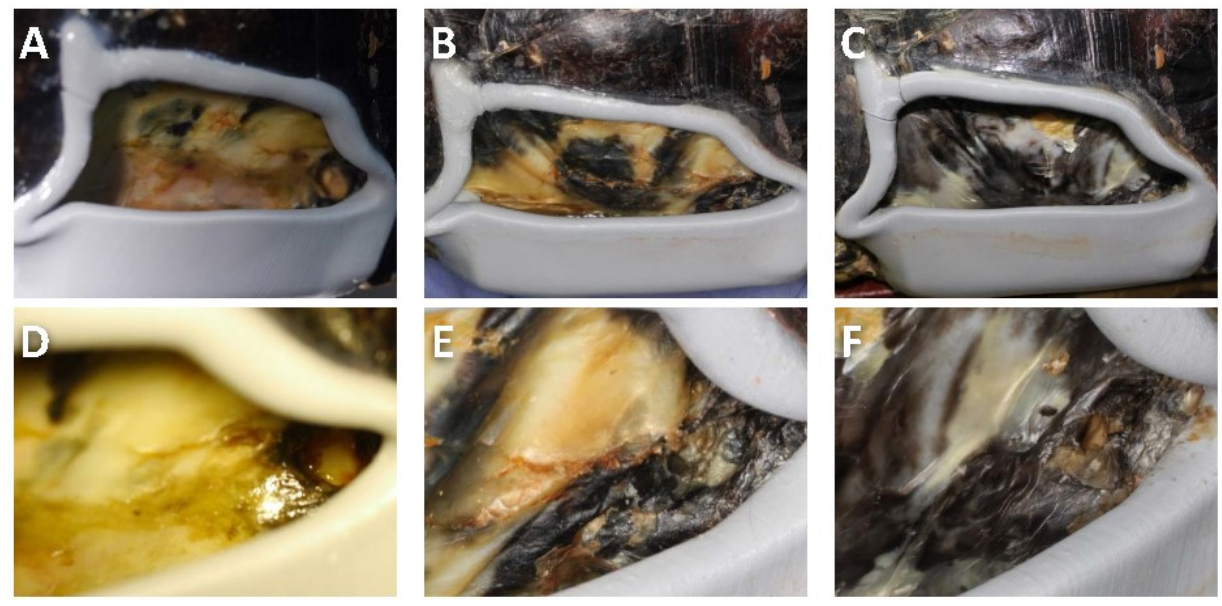

Figure 3. Clinical photos after devise application without cover structure: (A-C) lateral view; (D-F) direct view of soft tissue damage at the defect posterior area; (A) fragile tissue at Day 0; (B) small keratinised areas presentation at 3-months recall; $(C)$ mature keratinised tissue formation at 5-months recall; (D) fragile tissue with exudate formation at Day 0; (E) keratinised tissue formation at 3-months recall; (F) complete closure of fistula and mature keratinised tissue presentation at 5-months recall.
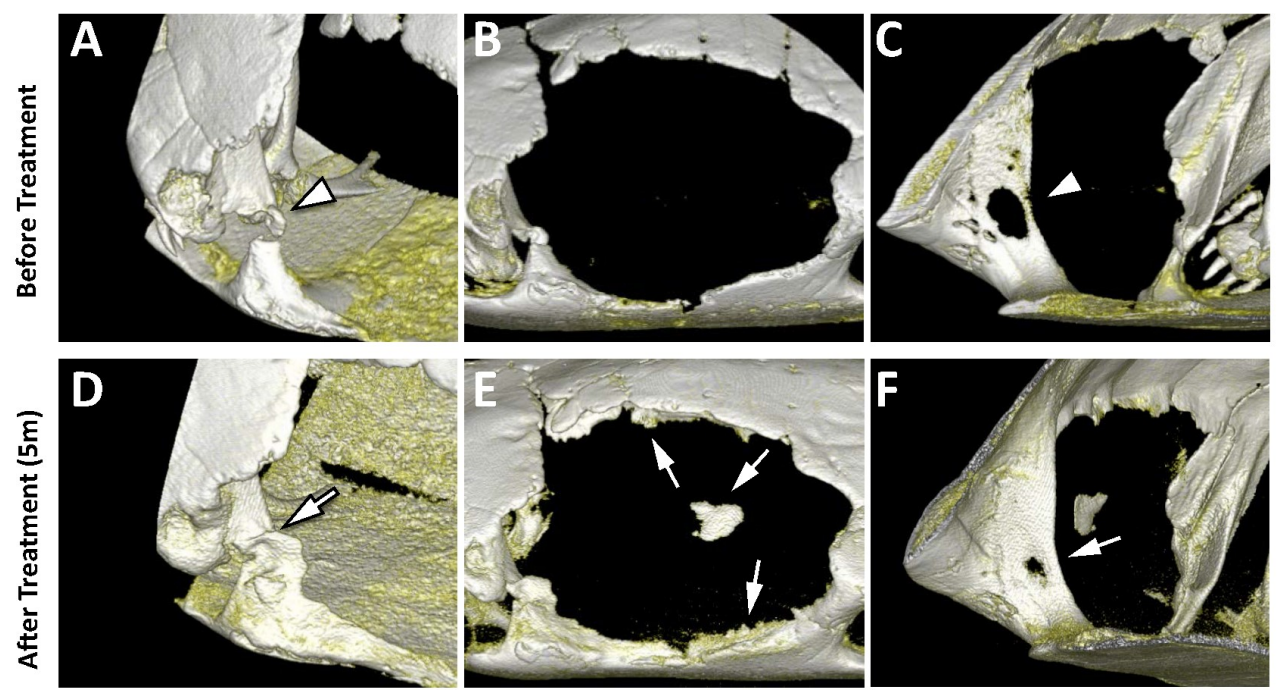

Figure 4. CBCT three-dimensional images of initial condition (A-C) and 5-month after devise application (D-F): (A) hard tissue defect on the anterior border (arrowhead); (B) defect lateral view; (C) hard tissue fenestration on the posterior border (arrowhead); (D) fracture healing (arrow) on the anterior border; (E) new hard tissue formation (arrow) at defect border and center area; (F) hard tissue healing (arrow) on the posterior border of the defect.

\section{Discussion}

One of the most common current issues encountered in wild freshwater turtles is shell injury. Most of these injuries were caused by road traffic accidents and predator attacks [7]. In this present report, we described the workflow of digital planning and 3D printed custom devices for a poor prognostic freshwater turtle with severe shell damage. At 12 months follow-up, the favorable outcome suggested that this shell-type device provides the protective ability and precisely fits the defect border with complex geometry. Several clinical reports demonstrated the treatment of shell injury of the freshwater turtle using different appliances. In contrast, there is insufficient information on the healing outcomes. Compared to these studies, the present report characterized wound healing conditions by clinical and 3D radiographic findings. 
3D technology has become feasible and affordable in recent years. The powerful machines become facile and easy to maintain, while the consumables material provides sustainable strength easy storage at a reasonable price[12]. This technology has been utilized in many fields of medication with favorable outcomes[13]. This report applied 3D scanning, CAD software, and 3D printing to successfully and rapidly achieve this rescue at a low cost. The 1-year follow-up result further consolidates the achievement of our treatment. These accessible 3D tools with high performance may provide an alternative way to help more animals with limited budget time and replace the human resource. With these 3D tools, the device's design can evolve fast along with the need after every test and discussion. The power of CAD software and printer gives us the ability to respond to needs and validate ideas rapidly..

The shell material and structure are an integrated system, which provides the loadbearing capacity and protecting ability from injury of the internal organs [14]. A previous study analyzed the stress distribution of turtle shells using a finite element model [15]. The highest value was located on the inside surface of the four bridges of the turtle shell. The initial 3D imaging showed that the left anterior bridge was fractured (Fig. 4A). Therefore, the device's material should provide strong resistance to the turtle shell. The PLA was selected as a leading candidate due to its thermoplastic and high-strength mechanical properties [16]. At a five-month examination, the hard tissue healing generally was observed radiographically at the anterior bridge of defect after application of the device (Fig. 4D). To make a firm attachment of the hull structure on the defect, we designed the snapin joints [17] in the groove to engage the overhanging shell. Compared with that conventional screw fixation, this non-invasive approach exempts anesthesia complications and the risk of infection failure. At the same time, the animal was weakened, and the bone defect was not stable. Therefore, we considered some sealants to fill the frame and the shell seam. PDMS was chosen for this purpose. PDMS is a biocompatible material that has been used in many medical devices [18]. Due to the excellent conformability, PDMS is broadly used in the semiconductor industry to replicate surface features down to subnano scale [19], and also commercially available as a sealant. With the property of biocompatibility and resistant to biological fouling [20], which is essential when the device is constantly in water, PDMS becomes the adequate sealant to eliminate the risk of infection and chemical toxicity [21].

We framed the defect with a hull. The hull design enhanced the framework's strength to compensate for the weakened shell structure and acted as a watertight armor to protect either vulnerable coelomic membrane or the clean environment from the contagious but inevitable water immersion. The hull is slightly above the waterline and left with a large opening for the following wound management (Fig. 2C and D). The opening provided a room to reach all the wounds inside the defect. The opening with an irregular frame was enclosed by the corresponding cover with a porthole (Fig. 2E and F). The cover was designed as a removable part when accessing the fundamental defect.

The porthole on the cover was designed and fabricated with thread that matched the wide bottle cap (Fig. 1F) available in the convenience store locally. As a result, the porthole can be neatly opened and closed, and the purulent pocket wound can be easily accessed through the porthole for daily care. In addition, this design avoided the frequent disengagement between the cover and hull, which further prevents the failure of watertight of the whole structure. The daily care was manageable with one medical personnel, and the workforce significasntly reduced with this device.

Although, the chelonian shell was still not fully recovered in 1-year follow-up. However, the CBCT examination indicated that there was a significant improvement in wound healing of hard tissue (Figure 4). The physical condition of the turtle was stable, and it could walk smoothly without the remarkable restriction of movement.

\section{Conclusions}


This report ably demonstrates a rapid and practical process to rescue a severely injured wild freshwater turtle using advanced 3D technology with limited human resources and a relatively low-cost way. Due to the maturation of 3D technology, these 3D tools have become robust and affordable. These sophisticated tools give us the chance to extricate an animal from the risk of euthanasia. This progression delivered an alternative point of view to apply the 3D technology on the wild animal rescue, either the whole veterinary medicine, which may help us to approach new way from the conventional practice.

Author Contributions: T. F. H., P. J. K and Y. S. N. organized the experimental plan. T. F. H. and P. J. K mainly carried out the experiments. T. F. H., P. J. K and Y. S. N. drafted of the manuscript. All authors read and approved the final manuscript.

Informed Consent Statement: In this case report, the wild animal was rescued under emergency situation in a veterinary hospital and was not subject to an experimental animal study. This report denotes a concept of 3D technology application in wild animal rescue by describing an alternative procedure that indeed extricates a wild animal from the situation of euthanasia. From an auxiliary approach, all the methods applied on the patient were non-invasive and designed diminishing any complication, while all the medications were performed by researcher who is licensed veterinarians in Taiwan.

Data Availability Statement: Not applicable".

Conflicts of Interest: The authors declare that they have no competing interests.

\section{References}

1. Hoang, D., et al., Surgical applications of three-dimensional printing: a review of the current literature E how to get started. Ann Transl Med, 2016. 4(23): p. 456.

2. Ma, L., et al., 3D-printed guiding templates for improved osteosarcoma resection. Sci Rep, 2016. 6: p. 23335.

3. Tack, P., et al., 3D-printing techniques in a medical setting: a systematic literature review. Biomed Eng Online, $2016.15(1)$ : p. 115.

4. Bauermeister, A.J., A. Zuriarrain, and M.I. Newman, Three-Dimensional Printing in Plastic and Reconstructive Surgery: A Systematic Review. Ann Plast Surg, 2016. 77(5): p. 569-576.

5. Vella, D., Management of aquatic turtle shell fractures. Lab Anim (NY), 2009. 38(2): p. 52-3.

6. Vella, D., Management of freshwater turtle shell injuries. Lab Anim (NY), 2009. 38(1): p. 13-4.

7. Fleming, G., Clinical Technique: Chelonian Shell Repair. Journal of Exotic Pet Medicine, 2008. 17(4): p. $246-258$.

8. Barten, S., Shell Damage, in Reptile medicine and surgery, S. Divers and D. Mader, Editors. 2005, Elsevier Saunders: USA.

9. J., K., et al., Chelonian Shell Fracture Repair Tecinques. Exotic DVM, 2001. 3: p. 35-41.

10. Hernandez-Divers, J.S., Surgery: Principles and Techniques, in BSAVA Manual of Reptiles, S.J. Girling and P. Raiti Editors. 2004, WILEY.

11. McArthur, S. and S. Hernandez-Divers, Surgery, in Medicine and Surgery of Tortoises and Turtles, S. McArthur, R. Wilkinson, and J. Meyer, Editors. 2008, Blackwell. p. 403-463.

12. He, Y., G.H. Xue, and J.Z. Fu, Fabrication of low cost soft tissue prostheses with the desktop 3D printer. Sci Rep, 2014.4 : p. 6973.

13. Ventola, C.L., Medical Applications for 3D Printing: Current and Projected Uses. P T, 2014. 39(10): p. 704-11.

14. Cebra-Thomas, J., et al., How the turtle forms its shell: a paracrine hypothesis of carapace formation. J Exp Zool B Mol Dev Evol, 2005. 304(6): p. 558-69.

15. Zhang, W., et al., Numerical Study of the Mechanical Response of Turtle Shell. Journal of Bionic Engineering, 2012. 9(3): p. $330-335$.

16. Farah, S., D.G. Anderson, and R. Langer, Physical and mechanical properties of PLA, and their functions in widespread applications A comprehensive review. Adv Drug Deliv Rev, 2016. 107: p. 367-392.

17. Klahn, C., D. Singer, and M. Meboldt, Design Guidelines for Additive Manufactured Snap-Fit Joints. Procedia CIRP, 2016. 50: p. 264269.

18. Tipnis, N.P. and D.J. Burgess, Sterilization of implantable polymer-based medical devices: A review. Int J Pharm, 2018. 544(2): p. 455460.

19. Teri W. Odom, et al., Improved Pattern Transfer in Soft Lithography Using Composite Stamps. Langmuir, 2002. 18(13): p. 5314-5320.

20. Ng, J.M., et al., Components for integrated poly (dimethylsiloxane) microfluidic systems. Electrophoresis, 2002. 23(20): p. $3461-73$.

21. Zhang, H. and M. Chiao, Anti-fouling Coatings of Poly(dimethylsiloxane) Devices for Biological and Biomedical Applications. J Med Biol Eng, 2015. 35(2): p. 143-155. 